\title{
Analysis of the Twitter Interactions during the Impeachment of Brazilian President
}

\author{
Fabrício Olivetti de França, \\ Denise Hideko Goya \\ Federal University of ABC, CMCC, \\ Nuvem Research Strategic Unit \\ folivetti@ufabc.edu.br,denise.goya@ufabc.edu.br
}

\author{
Claudio Luis de Camargo Penteado \\ Federal University of ABC, CECS, \\ Nuvem Research Strategic Unit \\ claudio.penteado@ufabc.edu.br
}

\begin{abstract}
The impeachment process that took place in Brazil on April, 2016, generated a large amount of posts on Internet Social Networks. These posts came from ordinary people, journalists, traditional and independent media, politicians and supporters. Interactions among users, by sharing news or opinions, can show the dynamics of communication inter and intra groups. This paper proposes a method for social networks interactions analysis by using motifs, frequent interactions patterns in network. This method is then applied to analyze data extracted from Twitter during the voting for the impeachment of the Brazilian president. Results of this analysis highlight the behavior of some users by retweeting each other to increase the importance of their opinion or to reach visibility. In addition, interaction patterns reveal that messages from one group (against/in favor of impeachment) rarely propagate to the opposing group. As such, this brings evidence that Social Networks may not stimulate a debate, but reaffirm users' beliefs.
\end{abstract}

\section{Introduction}

The outbreak of Arab Spring [1] drew attention to the political potential of Social Network Sites (SNS). Castells in [2] pointed out the emergence of a new model for political demonstrations that uses SNS to create a network of outrage and hope with the goal of articulating minds, create meaning and contest the institutional power.

Studies from different political demonstrations like Arab Spring, Indignados [3], and Occupy Wall Street [4] showed that the communication has an important role in the organizational structure of the demonstrations, thus emerging a connective action logic that characterizes by the use of SNS to promote personalized engagement [5].

Even though the connective action operates in a decentralized paradigm and leaderlessness, in [6] it was noticed the existence of Social Media Teams ("digital vanguards") the act like coordinators for the process of communication of digital accounts. As an example, during Occupy Wall Street, some users had a larger importance regarding the spreading of ideas acting as hubs [4] and playing a role of primary influential [7].

In [8] it was found that SNS like Facebook and Twitter has a positive effect on personal interactions and mobilization process, however, this medium also reinforces the distinction of different groups, allowing a rise of conflicts and hate speech, contributing to the lack of trust between groups.

Such a scenario promotes the occurrence of a social phenomenon known as homophily, defined as the tendency of similar people to form ties with each other, at a higher rate than among dissimilar people [9]. Homophily limits social worlds and the information received by an individual. Political content to which each user is exposed becomes restricted to its own points of view [10]. This natural restriction of the information flow within a specific group produces shared political attitudes which can result in political polarization [11, 12].

The authors of the last three cited works identified a formation of clusters by analyzing interactions networks on Twitter, involving posts in political themes. There were clusters in both followers networks $[10,11]$ and mentions network [12], with some evidence of homophily, although cross-ideological interactions between different clusters were observed, suggesting a coexistence of a public sphere, where a diversity of opinion can interact. In [11] was found higher levels of homophily in reciprocated followers network (where each relationship between a pair of users is symmetric) than in non-reciprocated network (with users being followed by those who does not follow them back).

Visibility is crucial to achieving symbolic power, defined as "the capacity to intervene in the course of 
events and influence the actions of others by means of the production and transmission of symbolic forms" [13]. In [14] it was showed that self-organized movement participants used strategies to leverage social media to better diffuse their message and enhance their symbolic power by combining the use of different hashtags to reach distinct social circles.

The work in [15] describes information cascade phenomenon, where there is an optimal point for an individual, by observing the choices of previous individuals, decides to ignore its own conflicting information and to adopt the trending idea. It is known that central users play a key role for information diffusion in SNS [16]. An interesting investigation would be to look for evidence of relations between centrality of users and their strategies to gain visibility and increase the adoption of their ideas.

\subsection{Case Context}

In June of 2013 Brazil witnessed a first wave of protests mobilized by the use of Internet social networks. These protests led millions of Brazilians to the streets requesting better public services and changes to the discredited democratic institutions [17].

A second wave of protests using SNS started in 2015 with demonstrations against corruption and demanding the impeachment of the just reelected Brazilian president, Dilma Rousseff [18, 19].

During the years of 2015 and 2106, groups in favor and against Rousseff's government promoted several political discussions on SNS [20].

In midst of an economic and political crisis, Brazilian lower house started the impeachment process on April 17th 2016, with 376 out of 511 of the congressmen votes in favor of this process.

This day had a great visibility in traditional media and an intensive usage of SNS by groups in favor and contrary to the impeachment. These groups tried to advocate their political position: the first commented several corruption scandals the president was accused of, and the second questioned the validity of such process and denounced that, in fact, a coup was in progress.

With the objective of understanding the contemporary public debate, more specifically in a strong political polarization context, we will study the debate between groups in favor and against the impeachment of the former Brazilian president Dilma Rousseff. As such, we pose two research questions:
RQ1. "During this specific event, do different groups create natural clusters preventing the information flow between different groups?"

RQ2. "Do central users create a pattern of interactions in order to reinforce their opinion and to strengthen their position in the dispute?"

This paper addresses these questions relative only to this specific event, while letting a full analysis for future research when data from different events are available.

In order to answer these questions, firstly it is established a measure for top influential users by the number of their interactions. After that, it is introduced a new method to understand the dynamics of these interactions by inspecting frequent patterns called motifs [21].

As a result, the contribution of this work is a new method to understand the interactions between groups of common and opposed opinions and some evidences toward how people make use of SNS environment to reinforce their opinions.

The paper is organized as follows: Section 2 gives a brief description for the adopted SNS and the performed procedures. In Section 3 the collected data set is analyzed, and in Section 4 are presented some final remarks and perspectives for future work.

\section{Methodology}

This section briefly describes the Twitter Social Network, highlighting concepts of interest for this research followed by the data collection method. After that, metrics of user importance are described and, finally, a new method for analyzing groups' interactions is proposed.

\subsection{Twitter Social Network}

Twitter Social Network [22] is a directed network with each node representing one user and the relationships modeled after a directional interaction between two users. Given two users, A and B, a directed edge from $\mathrm{A}$ to $\mathrm{B}$ means that $\mathrm{A}$ follows $\mathrm{B}$.

In this particular Social Network there is no need for a follower to be followed back, and thus nonreciprocated networks could be formed.

Every user is free to post a short message regarding any subject to be broadcasted to its followers (and, sometimes, the followers of its followers). The main interaction of this network is called retweet in which a 
given user broadcasts a message that was originally posted by someone else.

With this retweeting action, a given message can reach any user of network, without the restriction of having to follow the original poster.

\subsection{Data Collection}

The data collection comprehends the period of April 15 to April 19 of 2016. This period corresponds to two days prior and after the voting, respectively. For this purpose, the Twitter Streaming API (http://dev.twitter.com) provides a continuous flow of tweets limited to $1 \%$ of the total amount of tweets being published globally at every time step [23].

In order to narrow the results, the API provides a filter in order to retrieve only the tweets containing one of a set of keywords, limiting the total number being retrieved. Notice that, depending of the filter, this allows us to retrieve the entirety of the tweets pertaining to a given topic. For example, if $1 \%$ of tweets account for 1 million tweets and the applied filter retrieves 900 thousands tweets, then the API will provide the full set of requested tweets.

For this report, it was applied the content filter that retrieves only tweets containing one of the requested keywords. The filter is case-insensitive and replaces most accented character to its non-accented equivalents.
The keywords used in this work are listed in Table 1 and they are grouped as those commonly used by the group in favor of the impeachment (Pro), contrary to the impeachment (Con) and by both groups (Both). Notice that these terms were manually selected by observing the main active groups from each side. In Portuguese, words such as "Fora and "NuncaMais" means "Go away" and "Never more"; those were used to form hashtags used by groups in favor of the impeachment. The word "Golpe" means "coup" and was one of the main words adopted by users against the impeachment process.

During this period, it was collected 2,372,914 tweets from 503,181 different users containing at least one of these keywords. The tweets containing Pro or Con keywords were annotated as such, the tweets from the news accounts were classified as Neutral and the remainder was classified by using a Gradient Tree Boosting classifier [24] with a training procedure as devised in [18].

After that, we built a network of retweets relationship. In this network, each user is a node and edges from $\mathrm{A}$ to $\mathrm{B}$ means that user $\mathrm{A}$ retweeted a message from user B. Edges are weighted by the number of interactions between $\mathrm{A}$ and $\mathrm{B}$.

After discarding the users without any interaction, the generated network contained 371,509 nodes and $1,149,909$ edges and its giant component (i.e., the largest connected component of the network [25]) had 14,160 nodes and 200,877 edges.

Table 1. Terms used during the data collection on Twitter.

\begin{tabular}{|l|l|l|}
\hline \multicolumn{1}{|c|}{ Pro } & \multicolumn{1}{|c|}{ Con } & \multicolumn{1}{c|}{ Both } \\
\hline ImpeachmentJá, ForaDilma, & Golpe, NãoVaiTerGolpe, IstoÉGolpe, & Impeachment, \\
ForaLula, ForaPT, DilmaSai, & FicaDilma, DilmaFica, MidiaGolpista, & OBrasilNãoÉParaAmadores \\
SaiDilma, PTNuncaMais, & OcupaRedeEsgoto, Aeciomaiscitadoque, & , Polícia Federal, Delcídio, \\
LulaNuncaMais, RenunciaDilma, & DilmaMudaMais, ParaTiGlobo, & Congonhas, Condução \\
jesuijararaca, & LulaEstamosComVoce, LulaEuConfio, & Coercitiva, LulaMinistro, \\
somostodosmoro, ACasaTaCaindo, & SomosTodosLula, PovoComLula, Lula2018, & QuedaDoPlanalto, Catta \\
DiaHistorico, OpLavaJato, DeixaAP & LulaPresoPolítico, FechadoComOLula, & Preta, MortadelaDay \\
FTrabalhar, FimdaEraLula, Aletheia, & MoroExonerado, VemPraRua13Mar, & \\
OpAletheia, Lulanapapuda, & VemPraRua, 13MarEuVou, & \\
LulaPreso, EleNãoSabeDeNada, & 13MarVemPraRua, 13Mar, & \\
CalaBocaVcVotouNoPT & VemPraDemocracia & \\
\hline
\end{tabular}

\subsection{User Importance Measures}

In order to measure the importance of each user from this network, also called centrality [16], it was used importance measures based on the in and outdegree of each node.
In-degree centrality returns a value proportional to the in-degree of a node. In this network, this means how many retweets a user received. Likewise, Outdegree centrality is proportional to the out-degree, or how much a user retweets from others. 
Finally, it also was used PageRank [16] centrality in which a high value means that a user was retweeted from users with a high rate of retweets. In other words, in this measure an important person is one that connects with other important persons.

\subsection{Frequent RT Interactions}

A first inspection of this network revealed that some users presented an above average frequency of interaction when contrasted to most edges.

These frequent interactions occurred mostly between users with a high centrality, and always involving users with the same opinion. Another observation was that not all pairs of important users with the same opinion interacted frequently.

One suspicion was that those interactions might occur indirectly in order to avoid a perception of an organized group united for a cause.

In other words, users with a political agenda have as a primary goal to become an important actor within the network so people will trust their opinion. But, if they explicitly help each other to reach this goal, they will lose some credibility within their followers.

As such, one way to find evidences of these claims is to count the frequency of some interaction motifs in the studied network.

Motifs are frequent patterns in networks [21] that occur with an observed higher frequency than random patterns. They were already observed in ecology studies and studies of the World Wide Web [21].

Three interactions were searched inside this network: $\mathrm{A} \rightarrow \mathrm{B}$ (A retweeted often from $\mathrm{B}$ ), $\mathrm{A} \rightarrow \mathrm{B}$ $\rightarrow \mathrm{A}$ ( $\mathrm{A}$ and $\mathrm{B}$ retweeted frequently from each other), and $\mathrm{A} \rightarrow \mathrm{B} \rightarrow \mathrm{C} \rightarrow \mathrm{A}(\mathrm{A}, \mathrm{B}$ and $\mathrm{C}$ form a retweeting circle).

These frequent interactions were all searched through an enumerative process, and those with a frequency above than a specified threshold were marked as frequent.

\section{Results}

In this section are reported results from the analysis explained in the previous section along with some insights of what can be learned from them.

\subsection{Users Importance}

By using the giant component, it was extracted the top 10 users with each of centrality measures explained in Sec. 2.3. From Table 2, it is possible to count seven news related accounts in In-degree column, on the other hand, in Out-degree column none of them are related to news media. This seems reasonable since it is expected that news media produces information and gets retweeted more often and that personal accounts retweets and comments on information provided by news channels.

Since the PageRank measure favors those retweeted by users with lots of retweets, it was expected a predominance of news media, like in In-degree column. But, only three users related to news media were among the top 10 . So, somehow, some personal accounts could reach a higher degree of importance during this event.

Specifically in In-degree column, the users Estadao, JornalOGlobo and folha, are online accounts of traditional newspapers. In addition, BlogDoPim, GeorgeMarques and BlogdoNoblat are accounts from well known journalists, and MidiaNinja is an independent news source. Two users between these top 10 are apparently very popular teenagers who tells jokes about, but do not discuss politics. The only one in this list in a position clearly against the impeachment is ptbrasil, the official account of Dilma Rousseff's party.

From Out-degree column, br45ilnocorrupt, beijopai and Beamaral84 are accounts in favor of the impeachment. The other seven users with high outdegree are personal accounts against it.

Apart from news accounts, already noted, PageRank column contains personal accounts all in favor of the impeachment process.

Table 2. Top 10 users

\begin{tabular}{|r|l|l|l|}
\hline & \multicolumn{1}{|c|}{ In-degree } & \multicolumn{1}{|c|}{ Out-degree } & \multicolumn{1}{|c|}{ PageRank } \\
\hline 1 & Estadao & dionianjos & afpressuto \\
\hline 2 & luscas & beijopai & br45ilnocorrupt \\
\hline 3 & BlogDoPim & leleabreuv & BlogDoPim \\
\hline 4 & ptbrasil & moemasbc57 & MollerSandayo \\
\hline 5 & GeorgeMarques & Beamaral84 & lobaoeletrico \\
\hline 6 & JornalOGlobo & lacerdagalo & Estadao \\
\hline 7 & naosejatrouxa & woodstock_59 & diegoescosteguy \\
\hline 8 & BlogdoNoblat & araujosergio & BlogdoNoblat \\
\hline 9 & MidiaNinja & br45ilnocorrupt & MovBrasillivre \\
\hline 10 & folha & mariaap9413193 & mendoncafilho \\
\hline
\end{tabular}

Related to the first research question, we have also measured the Pearson correlation coefficient of the opinion between pairs of users that interacted with each other. The correlation coefficient can range from 1 to 1 , with -1 meaning a predominance of interactions between users with opposed opinions, +1 a predominance of interactions between users of the same opinion and 0 meaning that the interactions occur at random. 
The measured correlation was 0.36 implying a majority of interactions only with users sharing the same opinion. Notice that the interactions of a user classified as Pro or Con with a news media reduces the correlation value.

As such, this correlation gives us an initial evidence of a lack of interest in interacting with users bearing conflicting opinions.

\subsection{Frequent RT Interactions}

Regarding the frequent RT interactions, as described in Sec. 2.3, the next analysis will highlight those that occur at a much higher frequency in every considered pattern. In the next figures, we will depict these outliers. The number in the arrows is the frequency of the observed interaction during five days.

The first relationship analyzed, $\mathrm{A} \rightarrow \mathrm{B}$ (Figure 1), can be usually associated with the relationship of famous users and their fans. In this situation the fan will share everything the idol post. In general, this relationship occurs in an asymmetric way, forming a non-reciprocated network.

The most frequent relationship depicts the user Daniiel_Rodr, a young person engaged in spreading posts about politics and GeorgMarques, a journalist and public relations professional who covers news about the Brazilian Congress and politics. Daniiel_Rodr is a young person that spreads posts about politics; by further investigation, he and other five users intensively retweeted GeorgMarques's posts but seemingly as a fan/idol relationship. LupaNews is a fact-checking agency; it was retweeted by putscabeyo, a teenager that disseminates news in any subject. Afpressuto is an anti-communist militant; he was intensively retweeted by IIMPEACHMENT, an account that supports many other users against the Workers' Party (possibly the former is part of the maintainers of the latter account).
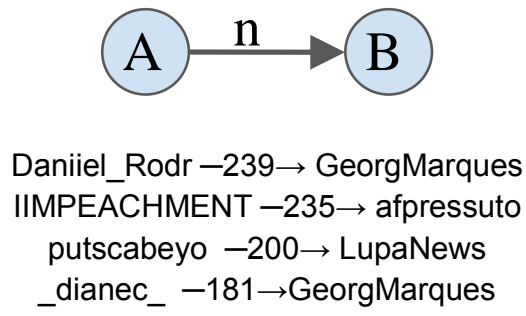

Figure 1. Frequent occurrences of $A \rightarrow B$ pattern, where $A-n \rightarrow B$ means that the user $A$ retweeted $n$ posts from the user $B$, in five days.
Finally,_dianec_account is currently deactivated, probably a fake account used to share news towards one opinion.

Next, the $\mathrm{A} \rightarrow \mathrm{B} \rightarrow \mathrm{A}$ pattern usually means two users who are friends to each other, and sharing the same opinion, retweet posts from each other. Some notable examples out of 145 collected are listed in Figure 2.

From this figure, the most frequent interaction found was between lobaoeletrico, a famous Brazilian artist in favor of the impeachment and br45silnocorrupt, an account created by PSDB (the party that lost the previous election) to engage people into protesting for the impeachment. This particular interaction brings some evidence for the second research question, in which users with common opinion reinforce each other their opinion in order to raise their authority.

The next two interactions are just retweeting Bots that shares random content from specific users and tweets text with popular hashtags to get more retweets.

Finally, the last frequent interaction was between two accounts of the PSDB party (that is assigned to number 45 in Brazilian elections). This particular interaction is not unexpected since they are both controlled by the same organization.

A more complex interaction pattern was also found in this network: $\mathrm{A} \rightarrow \mathrm{B} \rightarrow \mathrm{C} \rightarrow \mathrm{A}$. This pattern is more elaborate than those previously analyzed since it increases the distance between involved users.

Figure 3 shows three notable examples of such pattern. The first pattern was an extension to a previous observed interaction between lobaleletrico and br45ilnocorrupt with the inclusion of Cris_duh_123 a common person that mostly retweets news against the Workers' Party.

The next two interactions are among users that are self-proclaimed militants of the Workers' Party. Thought to a lesser extent, these interactions are still unexpected to occur at random.

Users that participated in these observed interactions were used to extract a subgraph of the interaction Graph. This created a graph with 169 nodes and 1,173 edges.

This network is depicted in Figure 4. In this figure, the colors represent the two communities found by the Label Propagation algorithm [16], with color red representing those supporting Dilma Rousseff and blue representing those against her. In green we highlighted accounts representing news media. It can be observed that green nodes are those who connect both communities, what is expected since they are used as a source of information by both sides. 


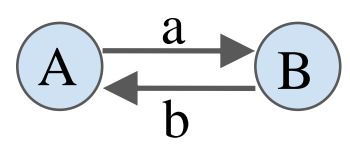

$$
\begin{aligned}
\text { lobaoeletrico }-62 & \rightarrow \text { br45ilnocorrupt }-37 \rightarrow \text { lobaoeletrico } \\
\text { kaicocavalcante }-42 & \rightarrow \text { timbetaramos } 28-46 \rightarrow \text { kaicocavalcante } \\
\text { nilton2cc }-9 & \rightarrow \text { DiogoBETASDV }-17 \rightarrow \text { nilton2cc } \\
\text { Rede } 45-6 & \rightarrow \text { PSDBnaCamara }-23 \rightarrow \text { Rede } 45
\end{aligned}
$$

Figure 2. Frequent occurrences of $\mathrm{A} \rightarrow \mathrm{B} \rightarrow \mathrm{A}$ pattern, where $a$ and $b$ represents the frequency.

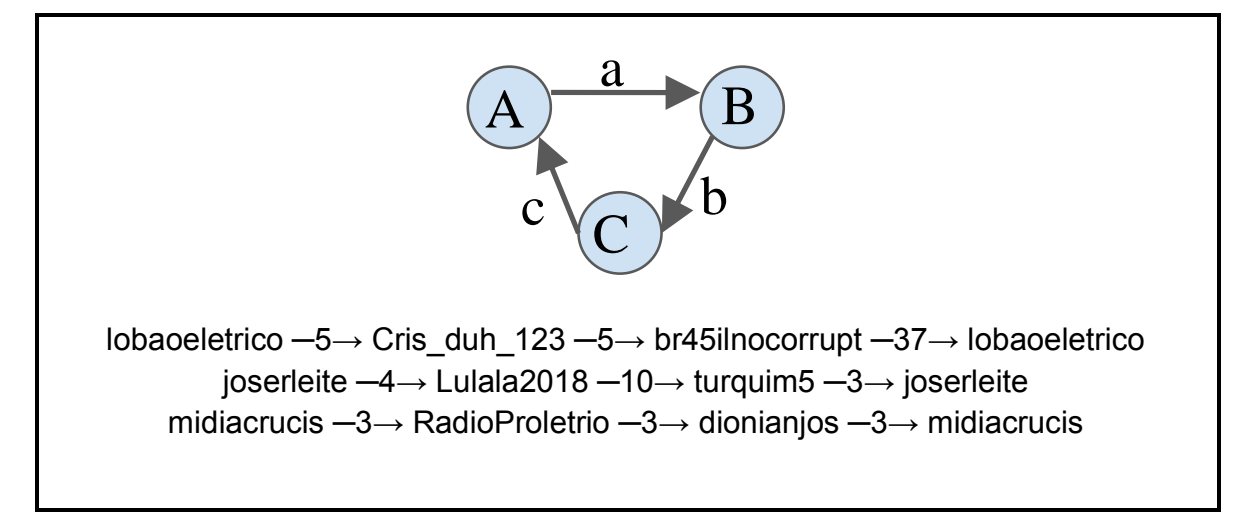

Figure 3. Frequent occurrences of $\mathrm{A} \rightarrow \mathrm{B} \rightarrow \mathrm{C} \rightarrow \mathrm{A}$ pattern, where $a, b$ and $c$ means frequency.

Notice that this network includes only $1.19 \%$ of the original sample (169 nodes out of 14,160) by including only those who interacted with another user more than three times, thus removing most casual users.

As such, this network comprehends only those users that could be involved in a debate concerning the impeachment. This brings evidence to both research questions posed in the beginning of this paper: i) there are groups with opposing opinions and they do not interact with each other, and ii) groups with similar opinion join forces to interact with each other.
This particular network with highlighted motifs brings a complementary result for the findings in [11] since there is a mix of non-reciprocated and reciprocated relationships forming a network with high level of homophily. And, unlike the works in [10, 11, 12], no cross-ideological interaction was found (i.e., no direct arcs linking a red to a blue point in Figure 4). 


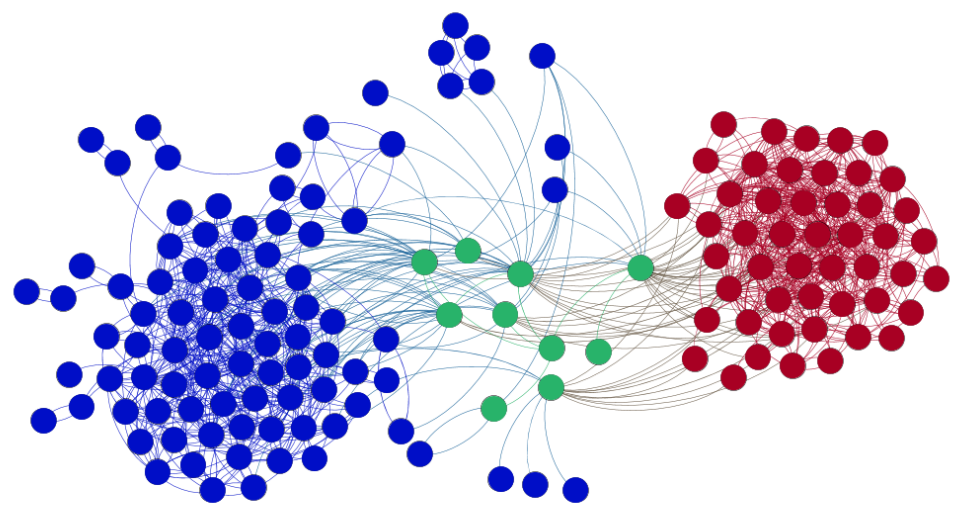

Figure 4. Network of the users with frequent retweeting interactions, forming patterns of 2 and 3 users that systematically retweet each other; the community in red is against the impeachment, the blue is pro, and between them are news media in green.

\section{Conclusion}

In this work, the interaction of users during online political debates was studied. Two clearly opposing groups were found, with evidences of high level of homophily in both of them, since the information flow was restricted within the originating group. Specifically, this paper focused on a data set extracted from the Twitter Social Network during the first vote for the impeachment process of the former Brazilian president Dilma Rousseff.

As such, we performed different Social Network Analysis measures and procedures in order to detect the most central actors, the tendency of preferential attachment and the communities created during the event. Additionally, we proposed a new analysis based on network motifs that revealed some interesting patterns of communication.

Regarding the actors centrality, we observed that the news media played an important role during the discussions by providing information in real time. Such information was used by both groups in order to feed their discussions. This shows that, despite a myriad of possible sources of information created by the Internet, users still trust the information provided by the traditional media.

Still regarding the centrality, we also observed the most predominant group when looking at the PageRank measure was those in favor of impeachment. One reason for this is the engagement of famous people and the creation of political groups with apparent intention of advocating against the former president. On the other hand, the group against the impeachment was predominant within the top outdegree users. One of the reasons for that was the coordinated action of this group to retweet a great amount of information denouncing a political intention for the impeachment.

When analyzing interactions between groups, it was found that the opposing sides did not interact with each other, signalizing a lack of proper discussion during the event.

Additionally, a community analysis of interaction network reveals a clear separation between these two groups, with news media acting as mediators.

These analyses answer positively our first Research Question, so in this particular event two much discerned groups were created preventing the information flow from one side to the other.

Regarding the motifs analysis, it was possible to find some interesting patterns within the network in which a chain of interactions is formed. This chain, if intentional, can help some users to increase their PageRank centrality with the after effect of increasing their visibility inside SNS. Also, this chain can create a false impression of validation of their own opinions, increasing the trust from the observers. These results answer positively our second Research Question regarding this event, since some central users was involved in these interaction patterns.

Even though SNS provides a freedom of speech and, thus, could create an environment for the discussion of ideas, influential users can be arranged in homophilic groups and may be applying artificial strategies in order to reach better visibility. 
Contrary to the libertarian spirit of creation of the Internet, as a free space for exchange and sharing of information where users can express their opinion, the creation of motifs in SNS may help to promote a segmentation of people with different beliefs.

For future work, we intend to replicate these experiments with different online discussions, not necessarily political related.

\section{Acknowledgements}

This work was supported by FAPESP-OSU-2015 Mobility Grant 2015/50250-9 and Nuvem Research Strategic Unit.

\section{References}

[1] P.N. Howard and M.M. Hussain, Democracy's fourth wave?: Digital media and the Arab Spring. Oxford University Press on Demand, 2013.

[2] M. Castells. Networks of outrage and hope: Social movements in the Internet age. John Wiley \& Sons, 2015.

[3] P. Gerbaudo. Tweets and the streets: Social media and contemporary activism. Pluto Press, 2012.

[4] M. Tremayne, "Anatomy of protest in the digital era: A network analysis of Twitter and Occupy Wall Street", Social Movement Studies 13.1, 2014, 110-126.

[5] W.L. Bennett and A. Segerberg, "The logic of connective action, Information", Communication \& Society, 15:5, 2012, p. 739-768.

[6] P. Gerbaudo, "Social media teams as digital vanguards: the question of leadership in the management of key Facebook and Twitter accounts of Occupy Wall Street, Indignados and UK Uncut", Information, Communication \& Society, vol. 20, n.2, 2016, 185-202.

[7] S. Gonzalez-Bailon, J. Borge-Holthoefer, and Y. Moreno, "Broadcasters and hidden influentials in online protest diffusion", American Behavioral Scientist, 57(7), 2013, p. 943-965.

[8] F. Sabatini and F. Sarracino, "E-Participation: Social Capital and the Internet", October 3, 2014. FEEM Working Paper No. 081.2014. Available at SSRN: https://ssrn.com/abstract=2505048 http://dx.doi.org/10.2139/ssrn.2505048

[9] M. McPherson, L. Smith-Lovin, and J.M. Cook, "Birds of a feather: Homophily in social networks", Annual Review of Sociology, 27, 2001, 415-444.

[10] I. Himelboim, S. McCreery, and M. Smith, "Birds of a Feather Tweet Together: Integrating Network and Content Analyses to Examine Cross-Ideology Exposure on Twitter", Journal of Computer-Mediated Communication, 18, 2013, 40-60.

[11] E. Colleoni, A. Rozza, and A. Arvidsson, "Echo chamber or public sphere? Predicting political orientation and measuring political homophily in Twitter using big data", Journal of Communication, 64.2, 2014, 317-332.
[12] A. Gruzd and J. Roy, "Investigating Political Polarization on Twitter: A Canadian Perspective". Policy \& Internet, 6, 2014, 28-45.

[13] J.B. Thompson, "The new visibility", Theory, Culture \& Society, Vol. 22 No. 6, 2005, pp. 31-51.

[14] R. Wang, W. Liu, and S. Gao, "Hashtags and information virality in networked social movement: Examining hashtag co-occurrence patterns", Online Information Review. Vol. 40 No. 7, 2016, pp. 850-866.

[15] S. Bikhchandani, D. Hirshleifer, and I. Welch, "A Theory of Fads, Fashion", Custom, and Cultural Exchange as Informational Cascades. Journal of Political Economy, 100, 1992, 992-1026.

[16] I. Pitas. Graph-Based Social Media Analysis. Chapman \& Hall/CRC, 2015.

[17] G. Cardoso, T. Lapa, and B. di Fátima. "People are the message? Social mobilization and social media in Brazil", International Journal of Communication, 2016, pp. 39093930 .

[18] C. de Souza Carvalho, F.O. de França, D.H. Goya, and C.L. de Camargo Penteado, "Brazilians divided: Political protests as told by twitter", In: Transactions on Large-Scale Data-and Knowledge-Centered Systems XXVII, Editors: Hameurlain, Küng, Wagner, Anjomshoaa, Hung, Kalisch, Sobolevsky, Springer, 2016, pp. 1-18.

[19] C. de Souza Carvalho, F.O. de França, D.H. Goya, and C.L. de Camargo Penteado, "The people have spoken: Conflicting Brazilian protests on Twitter". In: System Sciences (HICSS), 49th Hawaii International Conference on, IEEE, 2016, pp. 1986-1995.

[20] C.L. Penteado, and J.G. Guerbali. "As manifestações do impeachment no Twitter: uma análise sobre as manifestações de 2015", Ponto-e-Vírgula. Revista de Ciências Sociais. ISSN 1982-4807, 19, 2016.

[21] R. Milo, S. Shen-Orr, S. Itzkovitz, N. Kashtan, D. Chklovskii, and U. Alon, "Network Motifs: Simple Building Blocks of Complex Networks Science", Vol. 298, No. 5594. (25 October 2002), pp. 824-827.

[22] H. Kwak, C. Lee, H. Park, and S. Moon, "What is Twitter, a social network or a news media?", In: Proceedings of the 19th international conference on World wide web, ACM, 2010, pp. 591-600.

[23] K. Makice, Twitter API: Up and running - Learn how to build applications with the Twitter API. O'Reilly Media, 2009.

[24] J.H. Friedman. "Greedy function approximation: a gradient boosting machine." Annals of Statistics, vol. 29, 2000, pp. 1189-1232.

[25] M. Molloy, and B. Reed. "The size of the giant component of a random graph with a given degree sequence." Combinatorics, probability and computing 7.03, 1998, pp. 295-305. 